\title{
An effective color image segmentation approach using neutrosophic adaptive mean shift clustering
}

\author{
Yanhui Guo $^{\mathrm{a}, *}$, Abdulkadir Şengür ${ }^{\mathrm{b}}$, Yaman Akbulut ${ }^{\mathrm{b}}$, Abriel Shipley $^{\mathrm{c}}$ \\ a Department of Computer Science, University of Illinois at Springfield, Springfield, IL, USA \\ ${ }^{\mathrm{b}}$ Firat University, Technology Faculty, Electrical and Electronics Engineering Dept., Elazig, Turkey \\ ${ }^{\mathrm{c}}$ Department of the Office of the Vice Chancellor for Research, University of Illinois at Urbana-Champaign, Champaign, IL, USA
}

\section{A R T I C L E I N F O}

\section{Keywords:}

Color image segmentation

Mean shift clustering

Neutrosophic set

Indeterminate filter

\begin{abstract}
A B S T R A C T
Color image segmentation can be defined as dividing a color image into several disjoint, homogeneous, and meaningful regions based on the color information. This paper proposes an efficient segmentation algorithm for color images based on neutrosophic adaptive mean shift (NAMS) clustering. Firstly, an image is transformed in neutrosophic set and interpreted by three subsets: true, indeterminate, and false memberships. Then a filter is designed using indeterminacy membership value, and neighbors' features are employed to alleviate indeterminacy degree of image. A new mean shift clustering, improved by neutrosophic set, is employed to categorize the pixels into different groups whose bandwidth is determined by the indeterminacy values adaptively. At last, the segmentation is achieved using the clustering results. Various experiments have been conducted to verify the performance of the proposed approach. A published method was then employed to take comparison with the NAMS on clean, low contrast, and noisy images, respectively. The results demonstrate the NAMS method achieves better performances on both clean image and low contrast and noisy images.
\end{abstract}

\section{Introduction}

Image segmentation divides an image into homogenous, disjoint and meaningful regions which has been deduced as an important and challenging application in image processing community [1,2]. Many techniques have emerged for image segmentation [3-5]. These techniques generally use gray scale intensity, color information or texture information. Color is an important quantity which can be used to improve the image segmentation quality compared to the methods only using intensity. More segmentation approaches have been conducted on color images with demanding needs.

Various segmentation algorithms have been proposed in the last decade can be broadly categorized into three groups: feature based, region based, and graph based methods [5]. Feature based algorithms generally use the color or texture information to group the similar features into well separated clusters [6]. This separation issue is handled by a pre-defined distance measure. Clustering based image segmentation approaches are generally efficient. However, some of them do not consider the spatial information which causes undersegmentation. Region based segmentation methods are capable of preserving the edge or spatial information to produce more homogeneous and compact regions [7]. The watershed algorithm [8] is a popular region based segmentation approach. However, it might lead to over segmentation. This drawback can be eliminated by using a further merging procedure to construct more meaningful regions $[7,9]$. Graph based techniques are quite successful in image segmentation because these techniques generally fuse both feature and spatial information to produce more compact and well defined regions $[10,11]$. The graph based approaches define a graph whose vertex corresponds to a region and weight of edge is defined as the likelihood to segmentation. A graph is separated into components according to the cost function of vertices and edges. Graph based methods require high computation complexity disables them in use of real-time applications.

Mean shift (MS) is an iterative and robust clustering technique [12]. It seeks the local modes by shifting data to the average of its neighborhood. It has been applied to image filtering [13], video tracking [14] and image segmentation [15]. For image segmentation task, MS produces homogeneous areas by fusing the sufficiently close convergence points. Comaniciu et al. $[15,16]$ used the mean shift to segment color images and track objects. Ozertem et al. [17] combined the MS algorithm and the spectral clustering method to produce an effective segmentation method. Tao et al. [18] incorporated MS and shortest spanning tree for effective segmentation of the ships in

\footnotetext{
* Corresponding author

E-mail address: yanhui.guo@aggiemail.usu.edu (Y. Guo).
} 
Table 1

Detailed steps of the NAMS.

Input: Color image

Output: Segmented image

Step 1: Map each channel of an image to neutrosophic set;

Step 2: Convolute each channel using the indeterminacy filter;

Step 3: Compute the indeterminate values of the channels;

Step 4: Randomly select an ungroup pixel $P_{i}$ and compute the bandwidth $b w$ using its indeterminate value;

Step 5: Cluster the pixels into current group which have less distance than the bandwidth $b w$;

Step 6: Update the bandwidth $b w$ using the average value of the indeterminate values in current cluster;

Step 7: Go to Step 5 until the mean of the current clustering become unchanged;

Step 8: Go to Step 4 until all pixels are clustered;

Step 9: Separate the image using the clustering result.

infrared images. Tao et al. [5] combined MS with normal graph cut method to perform robust color image segmentation. Park et al. [19] proposed an algorithm based on Gaussian mixture model and adaptive MS algorithm. The MS algorithm was used to decide the number of Gaussian components. Another hybrid algorithm by Liu et al. [20] incorporated MS and ant clustering technique for efficient image segmentation. A deterministic searching method was developed by incorporating the multiple cues into MS algorithm and was applied on human tracking in color videos [21]. Ai et al. [22] examined incorporating the temporal characteristics of acquired functional magnetic resonance imaging data with mean shift clustering for functional magnetic resonance imaging analysis to enhance activation detections. Lai et al. [23] used MS for histogram equalization and determined a set of textured regions by using the density of edge concentration by using the MS abased approach. Ozden et al. [24] proposed an approach using low-level features incorporating color, spatial information and texture features in MS algorithm for segmentation.

Although MS algorithm is quite successful in image segmentation, it is necessary to determine a proper bandwidth value for the input image. This situation creates two different categories of MS based image segmentation approaches. In the first category, researchers use the MS method to initially partition images and then another segmentation scheme is produced based on some other methods such as graph cuts or clustering methods. In the second category, researchers investigate to improve the MS segmentation based on some adaptive MS methods. Therefore, in this paper, we introduce the neutrosophic set (NS) theory to MS algorithm to develop an adaptive MS clustering algorithm for color image segmentation. In the proposed method, we employ an improved NS based filter. Previous filters in NS domain, such as alpha-mean filter, make edge blur, and cause erroneous boundary segmentation. Moreover, the local spatial feature was not considered with the global features. To alleviate these drawbacks, we present an efficient segmentation algorithm based on neturosophic adaptive mean shift clustering (NAMS) for color images. Each color channel of image is transformed in neutrosophic set and interpreted using three membership subsets: true, indeterminate and false memberships. An indeterminacy filter implemented using indeterminacy memberships and neighbor features is utilized to alleviate the indeterminacy in the spatial domain. A new adaptive mean shift clustering is improved by determining the bandwidth using the indeterminacy values adaptively, and employed to identify the pixels into non-overlap groups.

The remained paper is organized as: Section 2 describes the proposed approach. Section 3 discusses experiments and results. The conclusions are conducted in Section 4.

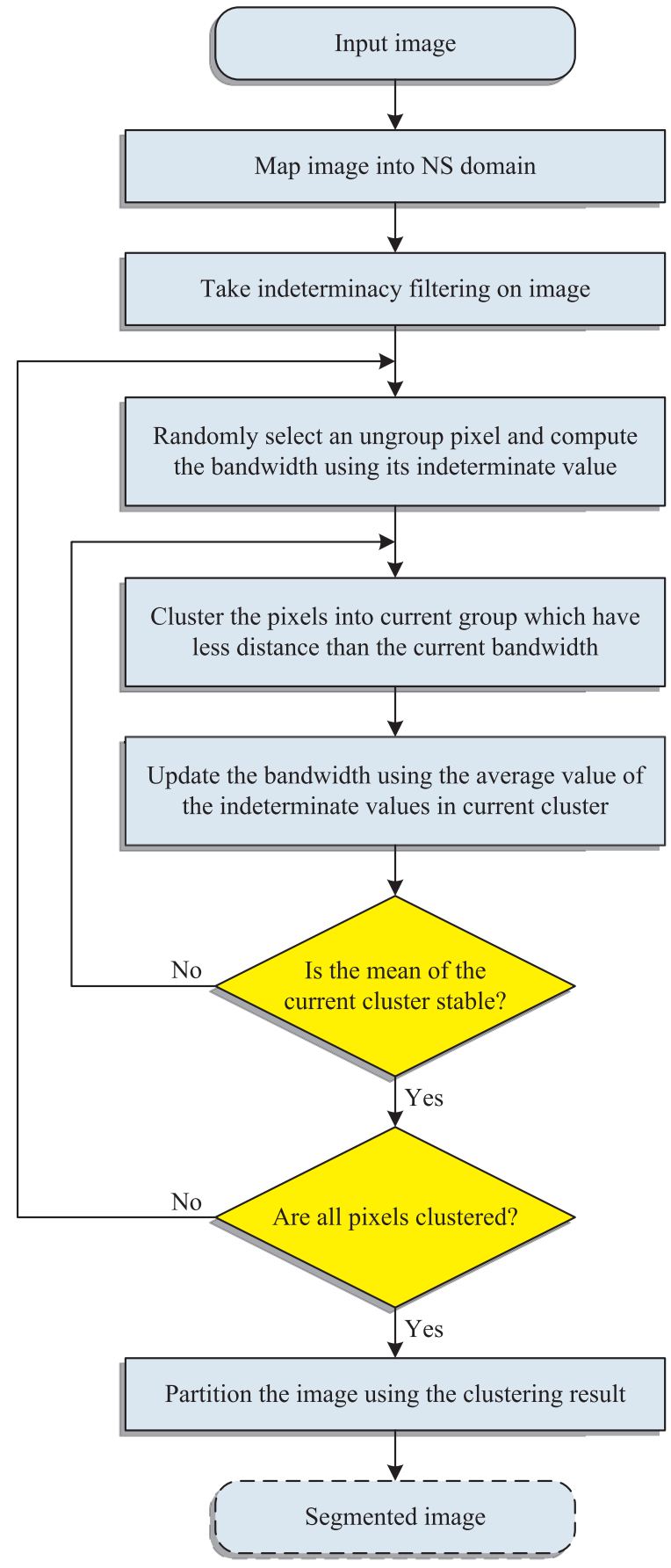

Fig. 1. The flowchart of the proposed method.

\section{Proposed method}

\subsection{Indeterminacy filter}

In this section, an indeterminacy filter is implemented to alleviate the disadvantages of traditional neutrosophic filter operations. Each color channel of an image is transformed in neutrsophic set, and a subset Is interprets each channel's indeterminate information on spatial domain. A filter named indeterminacy filter is constructed using Is to alleviate the indeterminacy of spatial information. 


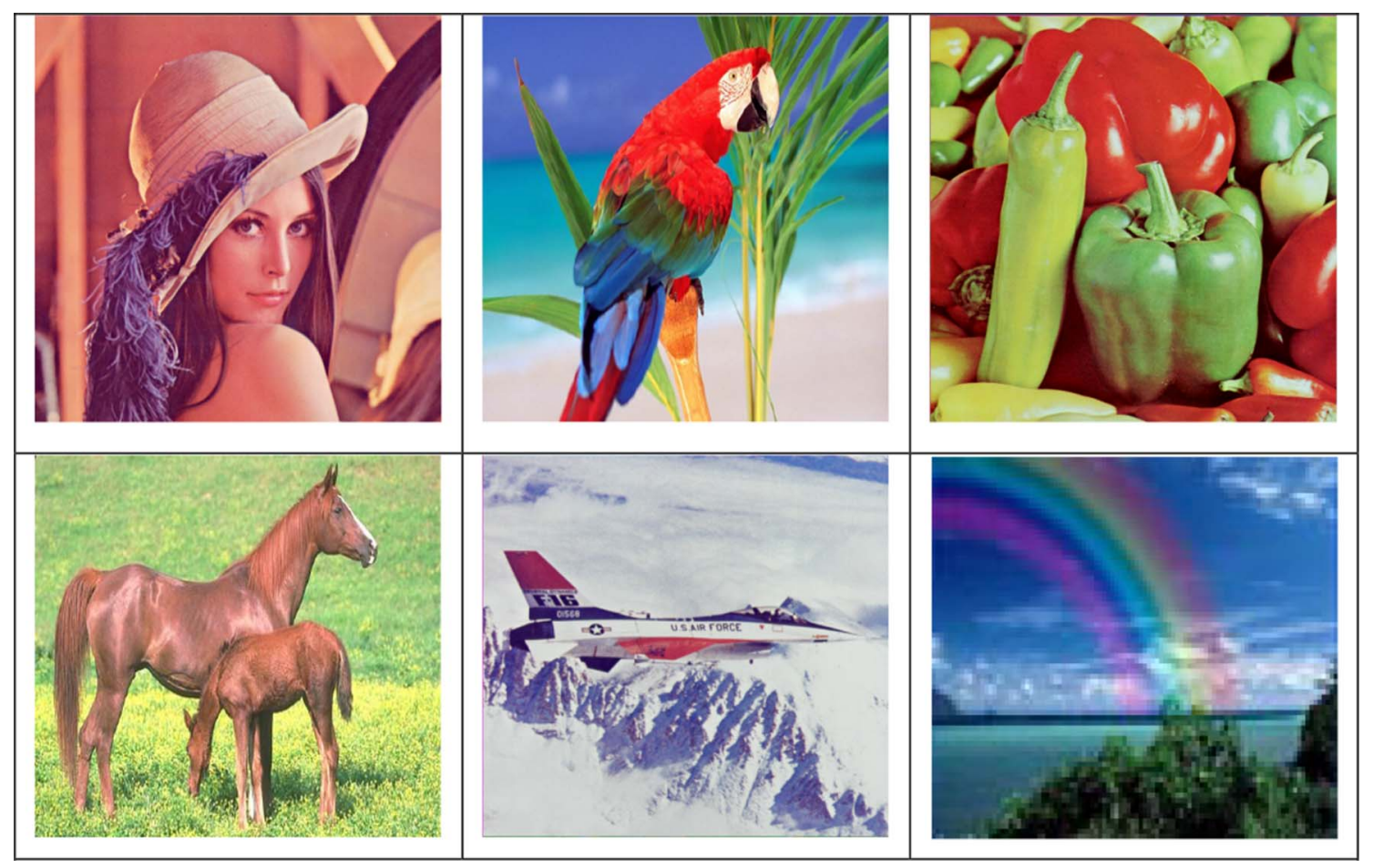

Fig. 2. Original natural images.

In neutrosophic set (NS), an alternative $A_{i}$ is denoted as $\left\{\mathrm{T}\left(A_{i}\right), \mathrm{I}\left(A_{i}\right), \mathrm{F}\left(A_{i}\right)\right\} / A_{i}$, where $\mathrm{T}\left(A_{i}\right), \mathrm{I}\left(A_{i}\right)$, and $\mathrm{F}\left(A_{i}\right)$ are denoted as memberships to the true, indeterminate and false sets, individually. A color channel in image $I C_{N S}$ is interpreted using three subsets $T_{C}, I_{C}$ and $F_{C}$. A pixel $P(x, y)$ in $I C_{N S}$ is denoted as $P_{N S}(\mathrm{x}, \mathrm{y})=\{\operatorname{Tc}(x, y), \operatorname{Ic}(x, y), \operatorname{Fc}(x, y)\} . \operatorname{Tc}(x, y), \operatorname{Ic}(x, y)$ and $\mathrm{Fc}(x, y)$ interpret the memberships to bright, indeterminate and black sets, respectively, which are defined using the intensity value as:

$\operatorname{Tc}(\mathrm{x}, \mathrm{y})=\frac{g(x, y)-g_{\text {min }}}{g_{\text {max }}-g_{\text {min }}}$

$\operatorname{Ic}(\mathrm{x}, \mathrm{y})=\frac{G d(x, y)-G d_{\min }}{G d_{\max }-G d_{\min }}$

$\mathrm{Fc}(\mathrm{x}, \mathrm{y})=\frac{g_{\max }-g(x, y)}{g_{\max }-g_{\min }}$

where $g(x, y)$ and $G d(x, y)$ are the intensity and gradient magnitude at $(x, y)$ on different color channels, respectively.

A filter is designed using the indeterminate value $\operatorname{Ic}(\mathrm{x}, \mathrm{y})$ and its kernel function is calculated as:

$G_{I c}(u, \mathrm{v})=\frac{1}{2 \pi \sigma_{I}^{2}} \exp \left(-\frac{u^{2}+v^{2}}{2 \sigma_{I}^{2}}\right)$

$\sigma_{I}(x, y)=f\left(I_{c}(x, y)\right)=a I_{c}(x, y)+b$

where $G_{I c}$ is a kernel function of the filter, and $\sigma_{I}$ is the standard deviation to determine the shape of the kernel function. A function $f(\cdot)$ is employed to map the indeterminate degree. When the indeterminate value is high, the value of $\sigma_{I}$ is large and the filtering can make the current pixel smooth according to neighbors. When it is low, the value of $\sigma_{I}$ is small and the filtering takes less smoothing operation. $\mathrm{a}$ and $\mathrm{b}$ are the parameters in the linear function to transform the indeterminate value to filter's parameter value.

A convolution is conducted on true membership value $\mathrm{Tc}(\mathrm{x}, \mathrm{y})$, and it will become more smooth after filtering.

$\mathrm{Tc}^{\prime}(\mathrm{x}, \mathrm{y})=\operatorname{Tc}(\mathrm{x}, \mathrm{y}) \oplus G_{I c}(\mathrm{u}, \mathrm{v})=\sum_{v=y-m / 2}^{y+m / 2} \sum_{u=x-m / 2}^{x+m / 2} T c(x-u, y-v) G_{I c}(\mathrm{u}, \mathrm{v})$

where $T_{c}^{\prime}$ is the result after indeterminate filter on $T c . G_{I c}$ is a kernel function of the filter, and $\mathrm{m}$ is its size.

\subsection{Mean shift}

Mean shift (MS) can find the maximum of a density function [12]. It has been applied into cluster analysis in image processing [15]. MS is defined as a scheme to find the maximum of a density function on discrete data [9]. It starts with an initial estimate $x$ and takes an iteration process. A kernel function $K\left(x_{i}-x\right)$ \} determines the weight of points to recalculate the mean. The weighted mean of the density is calculated as:

$\mathrm{m}(\mathrm{x})=\frac{\sum_{x_{i} \in N\left(x_{i}\right)} K\left(x_{i}-x\right) x_{i}}{\sum_{x_{i} \in N\left(x_{i}\right)} K\left(x_{i}-x\right)}$

where $N(x)$ is the neighbors of $x$ for which $K(x) \neq 0$.

The difference $m(x)-x$ is defined as mean shift [25]. The method sets $\mathrm{m}(\mathrm{x})$ to $\mathrm{x}$, and repeats the estimation until $\mathrm{m}(\mathrm{x})$ converges.

The result of mean shift clustering is controlled by the kernel bandwidth value. Other clustering approaches, such as k-means and fuzzy c-means, require a number of clusters to be specified as an input. At most time, the number of the clusters is unknown. It is an 


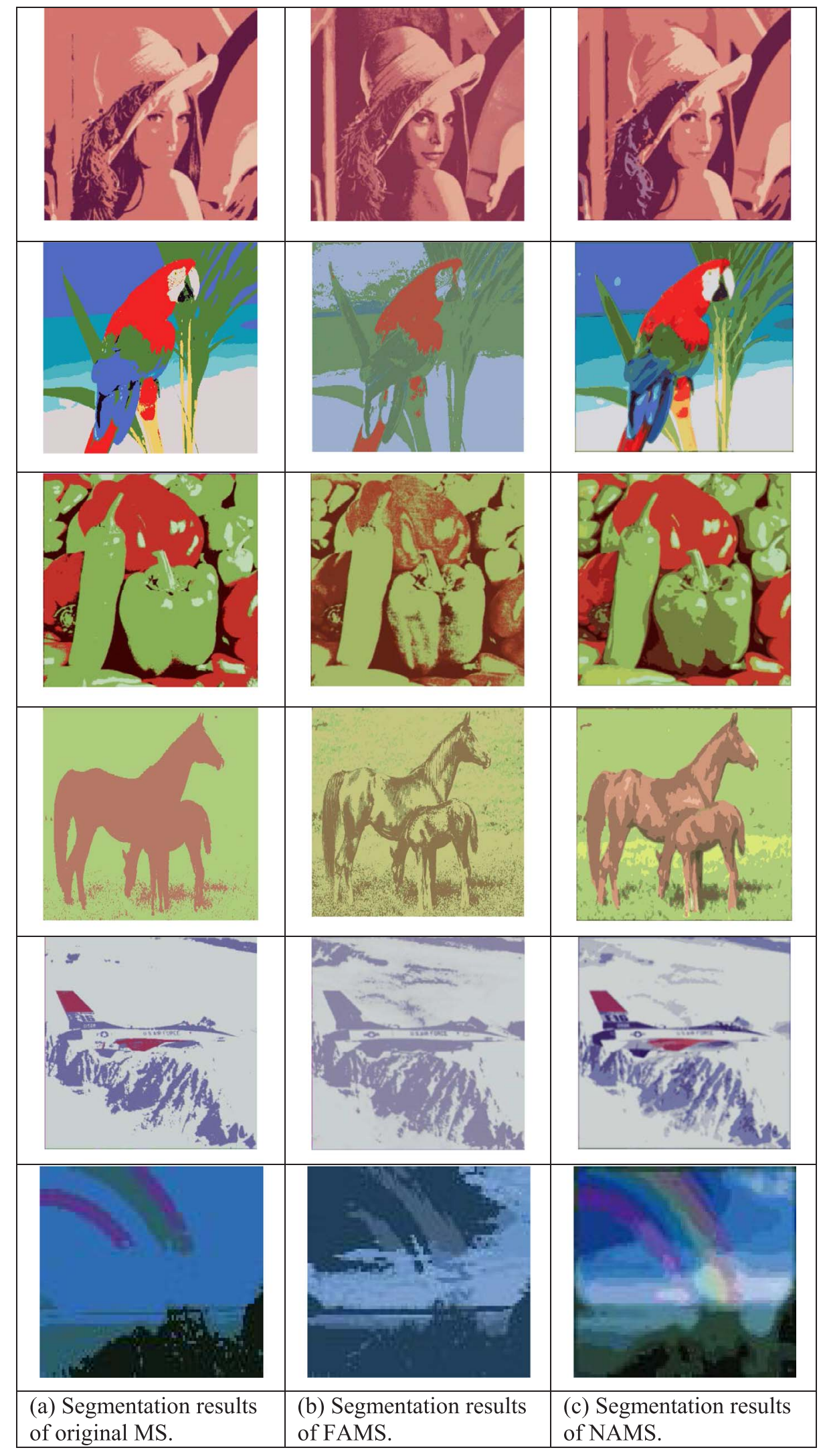

Fig. 3. Comparison of segmentation results on natural images. 


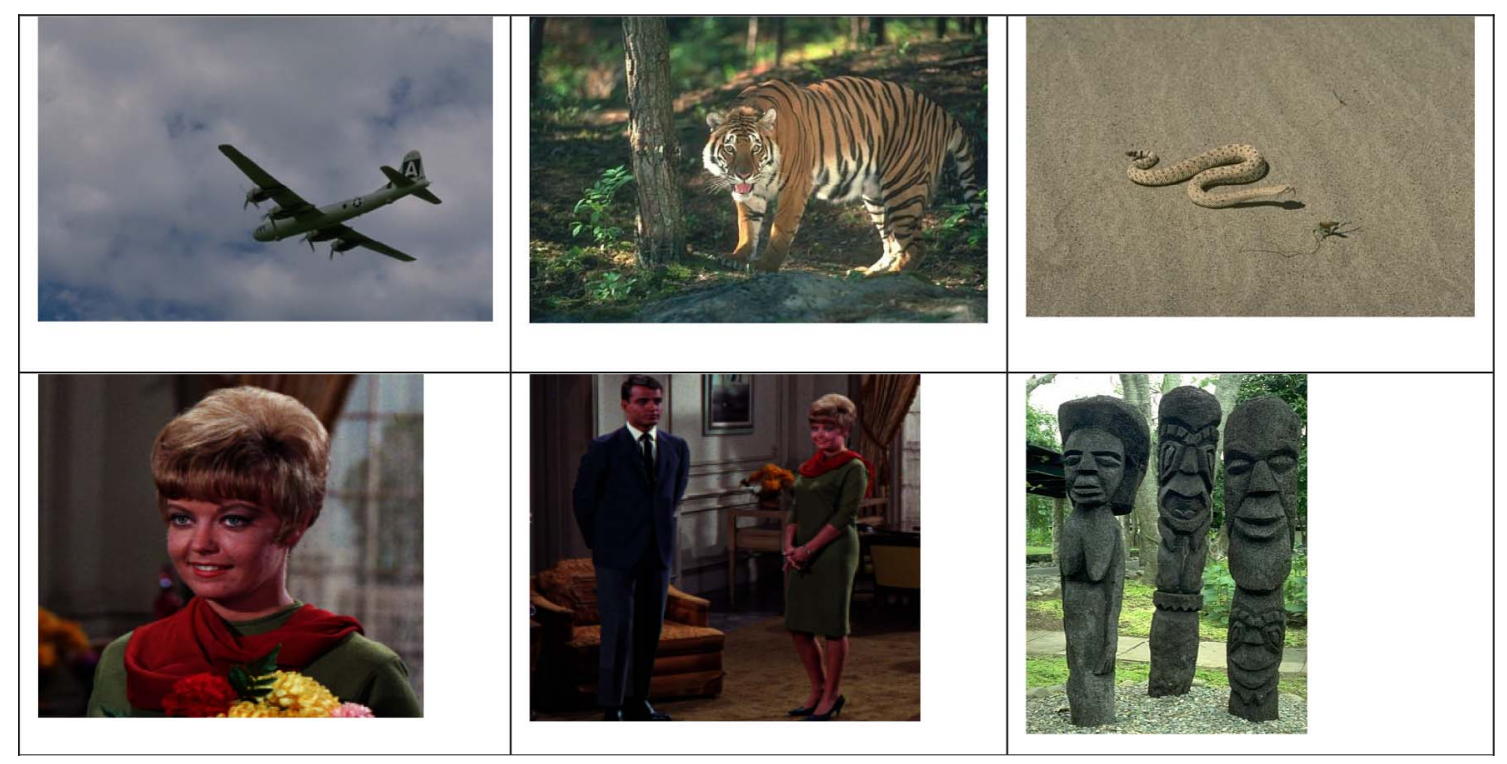

Fig. 4. Natural low contrast images.

important advantage of MS that it does not need the number of the clusters.

\subsection{Neutrosophic adaptive mean shift algorithm (NAMS)}

In the MS clustering, the value of kernel bandwidth can often be chosen based on some domain-specific knowledge. In the image segmentation problem, the bandwidth value can be viewed as how close colors need to be considered related to each other in color space. However, in most conditions, especially for image with different illumination situations, the kernel bandwidth is challenging to select and should be picked up adaptively according to image's local characteristics.

In the proposed method, the kernel bandwidth is determined using the indeterminate values adaptively.

$\mathrm{bw}(\mathrm{x}, \mathrm{y})=\mathrm{f}(\overline{\mathrm{Ic}}(\mathrm{x}, \mathrm{y}))$

where $\mathrm{bw}(\mathrm{x}, \mathrm{y})$ is the adaptive bandwidth of the current cluster, $\overline{\mathrm{Ic}}(x, y)$ is the average indeterminacy value of the current cluster at the center pixel $p(x, y)$, and $f(\cdot)$ is a function to map the indeterminate value to bandwidth value. In our algorithm, we use a linear function as:

$\mathrm{f}(\overline{\mathrm{Ic}}(\mathrm{x}, \mathrm{y}))=\overline{\mathrm{Ic}}(\mathrm{x}, \mathrm{y})\left(\mathrm{Tc}_{\text {max }}^{\prime}-\mathrm{Tc}_{\text {min }}^{\prime}\right)$

where $\mathrm{Tc}_{\text {max }}^{\prime}$ and $\mathrm{Tc}_{\text {min }}^{\prime}$ are the maximum and minimum of $\mathrm{Tc}^{\prime}$ at current cluster among all color channels.

All steps in the proposed NAMS method are summarized as follows (see Table 1):

The whole flowchart of the proposed NAMS method is described as Fig. 1.

\section{Experimental works and results}

The proposed NAMS has been tested on a variety of color images as shown in Fig. 2, and compared with a newly published fast adaptive mean shift (FAMS) color image segmentation algorithm [26] and traditional mean shift (MS) algorithm [15]. We follow the same parameters in the original papers. In the proposed method, all experiments are taken using the same parameters as: $\mathrm{a}=1$ and $\mathrm{b}=0.25$ which are obtained using trail-and-error technique.

\subsection{Performance on natural images}

Here, natural images are employed to validate the NAMS's performance which was commonly used in other publications. Six images in Fig. 2 are selected representatively to show the performance of the NAMS compared to other methods.

Fig. 3 shows segmentation results of MS, FAMS and NAMS methods for images. While the first column of Fig. 3 shows the MS segmentation results, second and third columns show the FAMS and proposed NAMS results, respectively. The results of "Lena" are shown in the first row of Fig. 3. The regions of the eyes, face, nose and mouth are preserved well in the third column. Areas above the hair and hat are also identified into correct group. The MS method takes incorrect segmentations in the area above hat. In the second row of the Fig. 3, there are segmentation results of the "Parrot" image. MS and the proposed NAMS produced almost similar segmentations while FAMS produced the worse segmentation. The head of the parrot is well segmented by the proposed NAMS method and the regions are quite homogenous. Similar comparing results are shown in the "Peppers" images in the third row of Fig. 3. When the proposed NAMS method result is implemented on the image, the surfaces of the pepper regions are segmented correctly and the results are consistent. On the other hand, these regions are corrupted by noise and results are worse in the MS and FAMS methods. The superiority of the NAMS algorithm can also be found in other images in Fig. 3. Thus, a general assumption can say that proposed NAMS shows better performance than those of FAMS and MS on visual. The results of the NAMS categorized most points into correct classes, while the boundary details are kept.

\subsection{Performance on low contrast images}

Some low contrast images are also used to evaluate the NAMS's performance. A comparison with MS and FAMS methods was also 


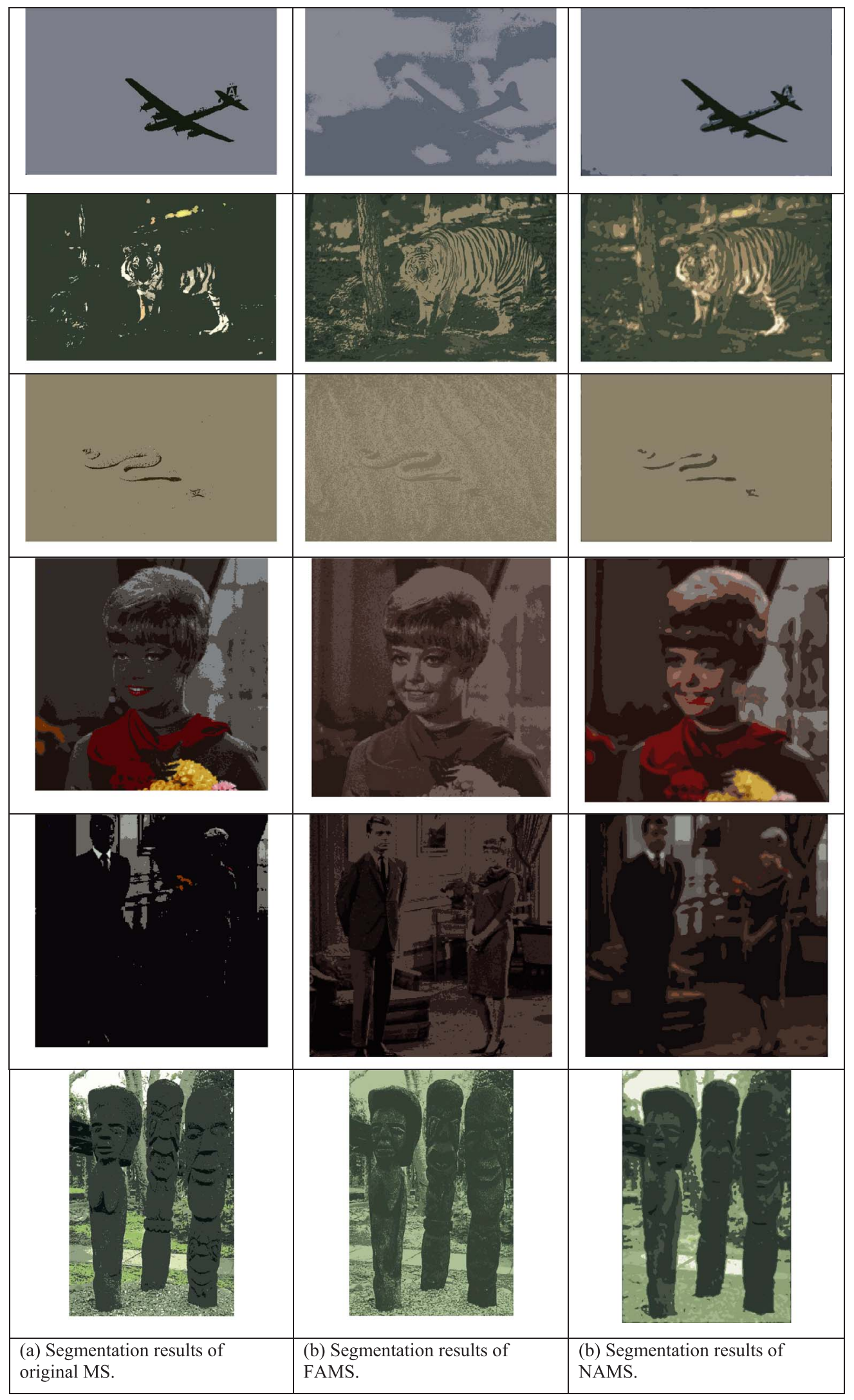

Fig. 5. Comparison of segmentation results on natural low contrast images. 


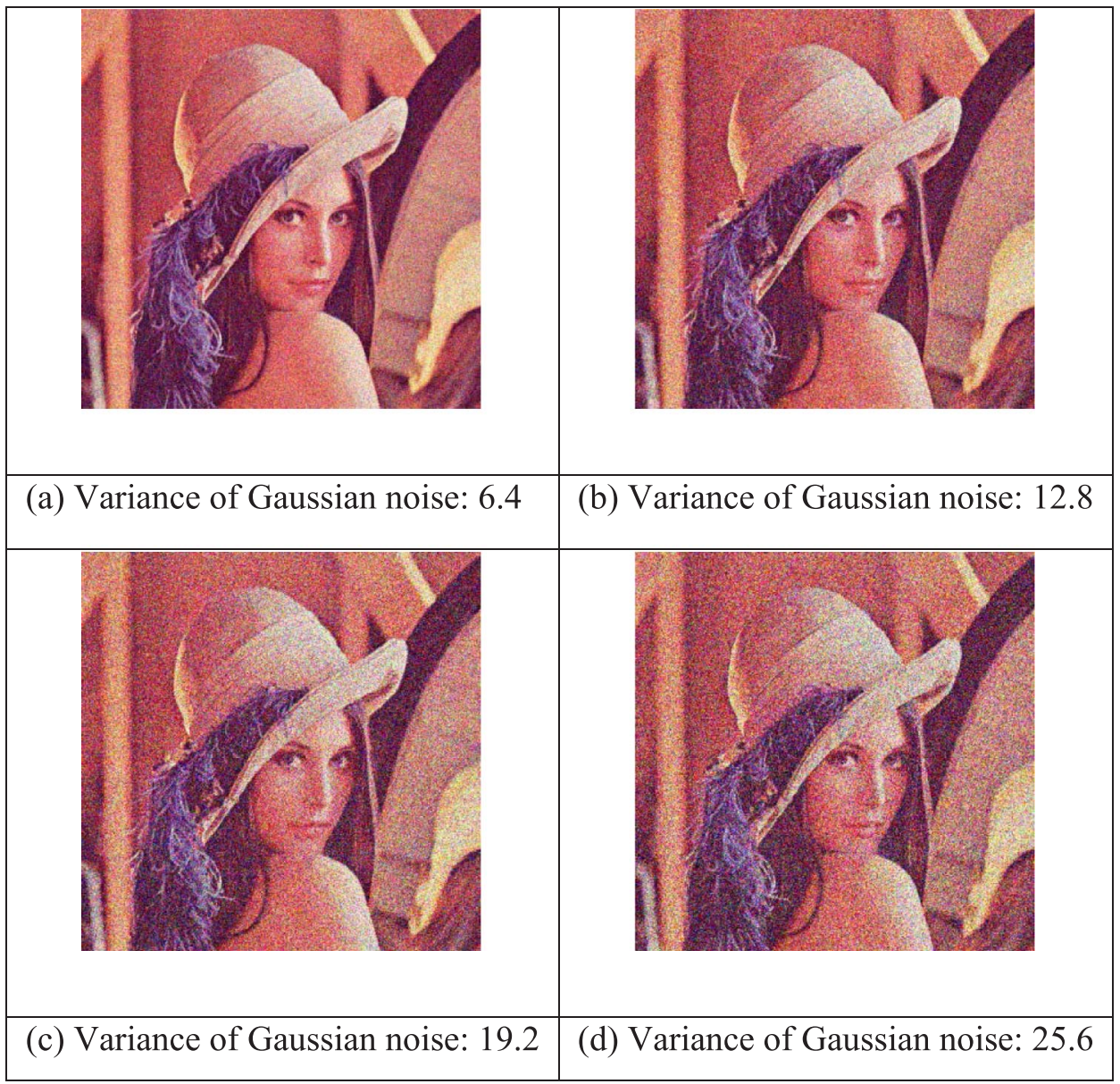

Fig. 6. Lena image with Gaussian noise.

considered on these images. The used low contrast images are shown in Fig.4 and results were given in Fig. 5, respectively.

The first row of Fig. 5 depicts the segmentation results on "Plane" image. It contains a plane in a cloudy sky with low contrast. Thus, the ground-truth segmentation should have two regions one for the plane and the other for background. When this assumption is considered, the worse result was produced by FAMS method where several cloud regions and plane were segmented as the same region. On the other hand, MS and proposed NAMS methods produced reasonable segmentations, where plane and sky regions were perfectly recovered. But, when MS segmentation result is analyzed in detail, it can be seen that some of the plane regions were segmented as background.

In the second row of Fig. 5, the results for "Tiger" image are given. As in Fig. 5, the MS method did not segment the regions on tiger correctly. In addition, the tree also segmented as background. FAMS also produced several false segmentations. The tiger and background regions were overlapped. In short, MS and FAMS methods did not produce meaningful regions. The proposed NAMS produced more proper regions. The tiger, tree and background regions are more meaningful. A snake image is used as it was given in the third row of Fig. 5. Similar to the previous results, MS and NAMS produced similar results while worse segmentation result was yielded by FAMS. In addition, MS segmentation result contained some noises on the snake region. In the rest results that were depicted in other rows of Fig. 5 proves that the proposed NAMS produced more meaningful and reasonable segmentation comparable to MS and FAMS methods.

\subsection{Performance on noisy images}

We also compared the segmentation performances of the proposed NAMS with MS and FAMS methods on noisy images. Figs. 6, 8, 10, and 12 show images corrupted by different noise levels. The Gaussian noise was added. The obtained segmentation results were given in Figs. 7, 9, 11 , and 13 , respectively. The results of NAMS were not affected by noises while the results of MS and FAMS were corrupted. In the comparing results, the proposed NAMS approach is well performed to separate noise free images and noisy images.

\section{Conclusion}

In this study, an efficient approach is proposed for color image using neutrosophic adaptive mean shift clustering method. An indeterminacy filter is implemented using the indeterminacy value and the spatial feature is utilized to alleviate the indeterminacy in the spatial domain. A new mean shift clustering, improved by neutrosophic set, is employed to categorize the pixels into different groups whose bandwidth is determined by the indeterminacy values adaptively. The segmentation results are achieved using the adaptive mean shift clustering results. A variety of experiments show the better performance of the NAMS approach than the existing algorithms because the indeterminacy has been depicted and employed well in the NAMS. The results also demonstrate the presented method is able to segment the images properly and effectively on both clean images and noisy images. 


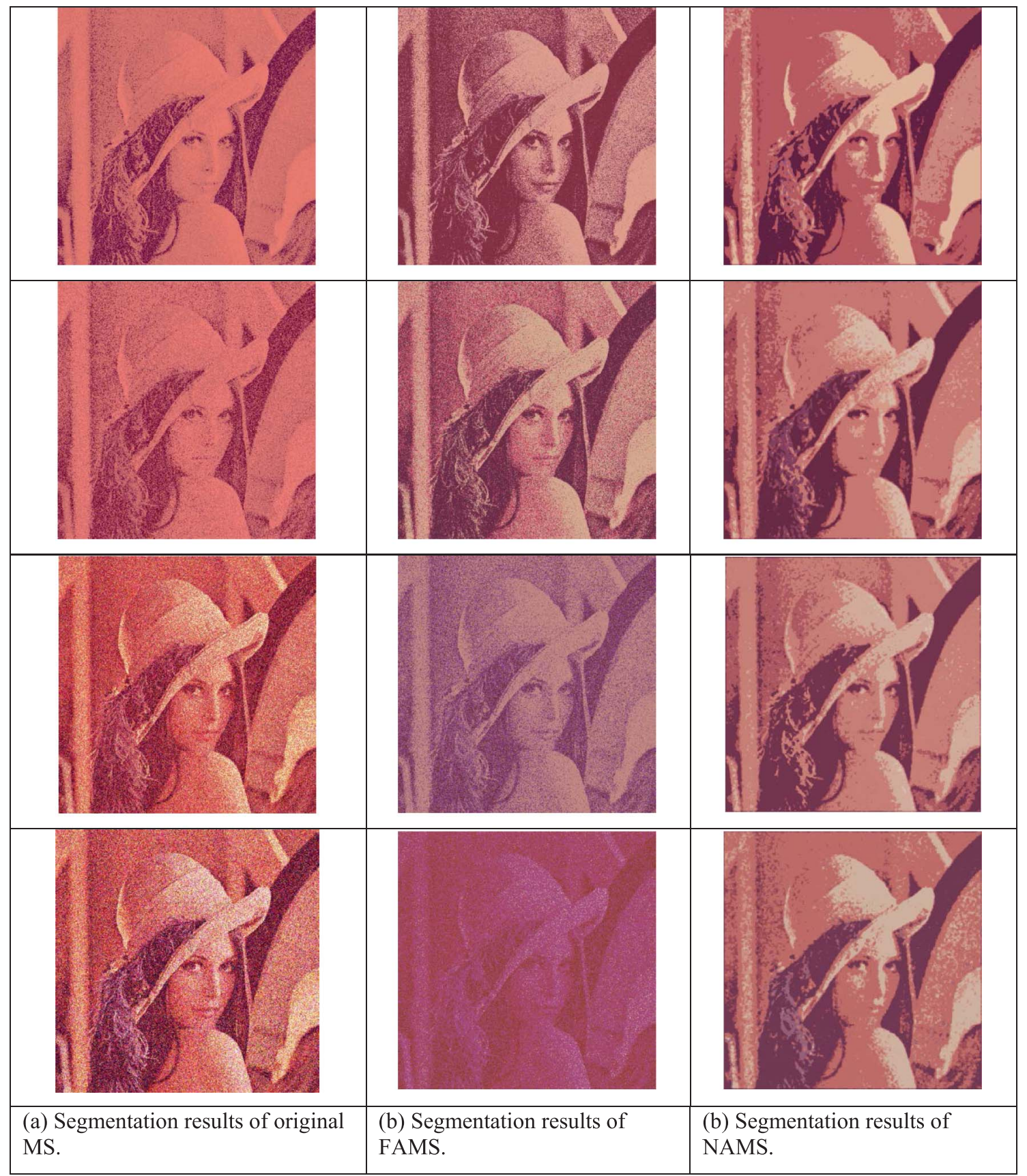

Fig. 7. Segmentation results on Lena image with Gaussian noise. 


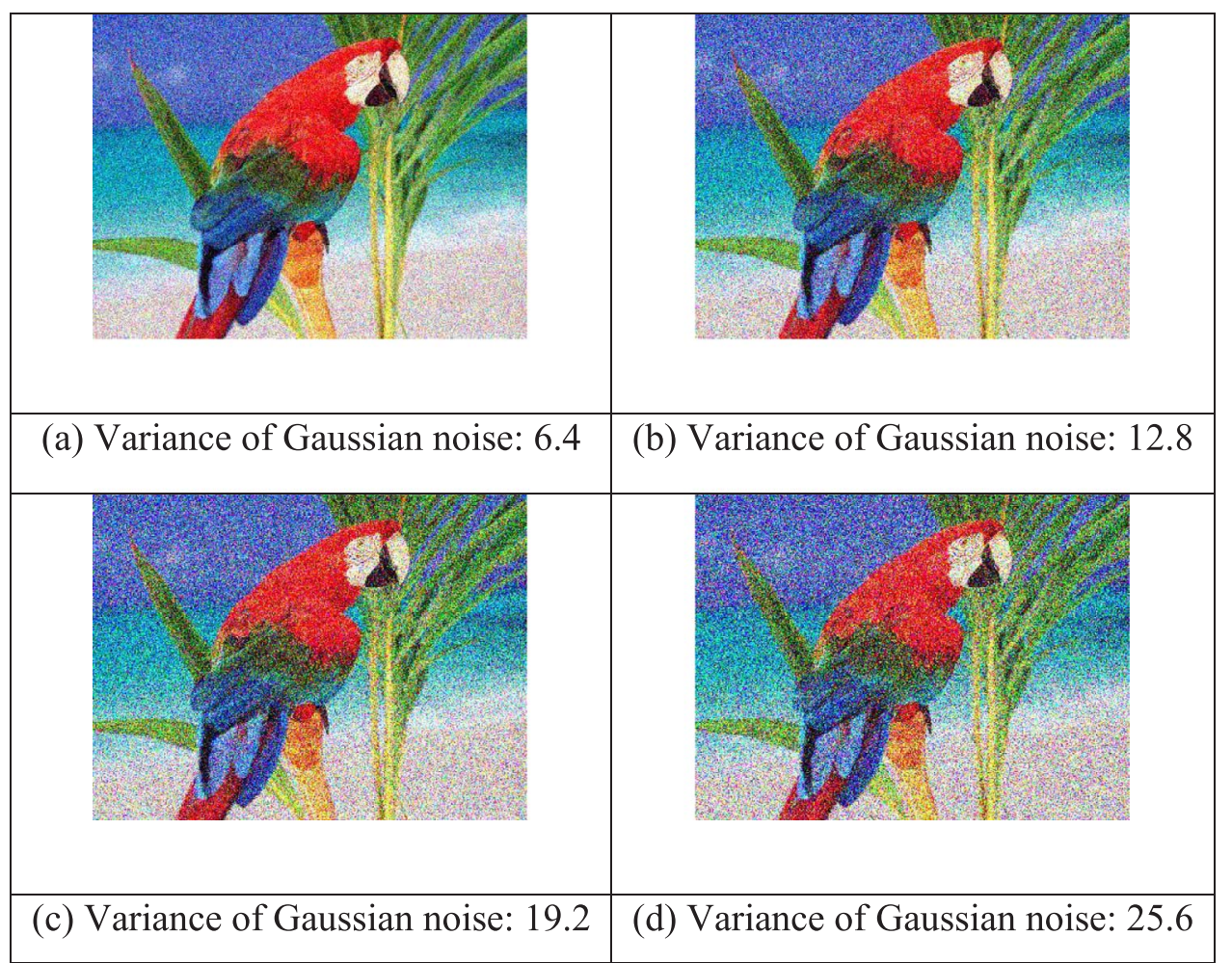

Fig. 8. Parrot image with Gaussian noise.

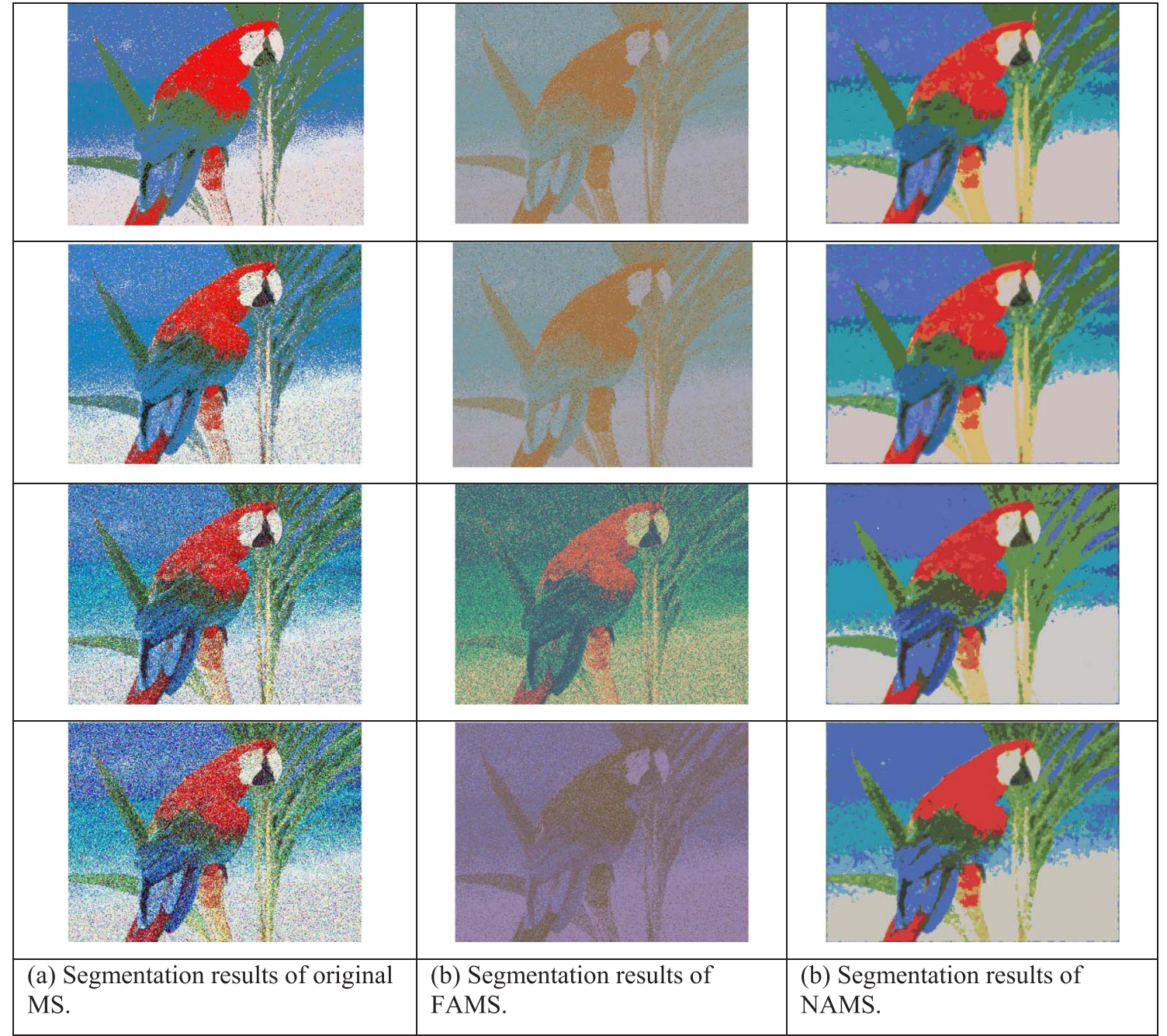

Fig. 9. Segmentation results on Parrot image with Gaussian noise. 


\begin{tabular}{|l|l|l|}
\hline 2 & \\
\hline
\end{tabular}

Fig. 10. Peppers image with Gaussian noise. 


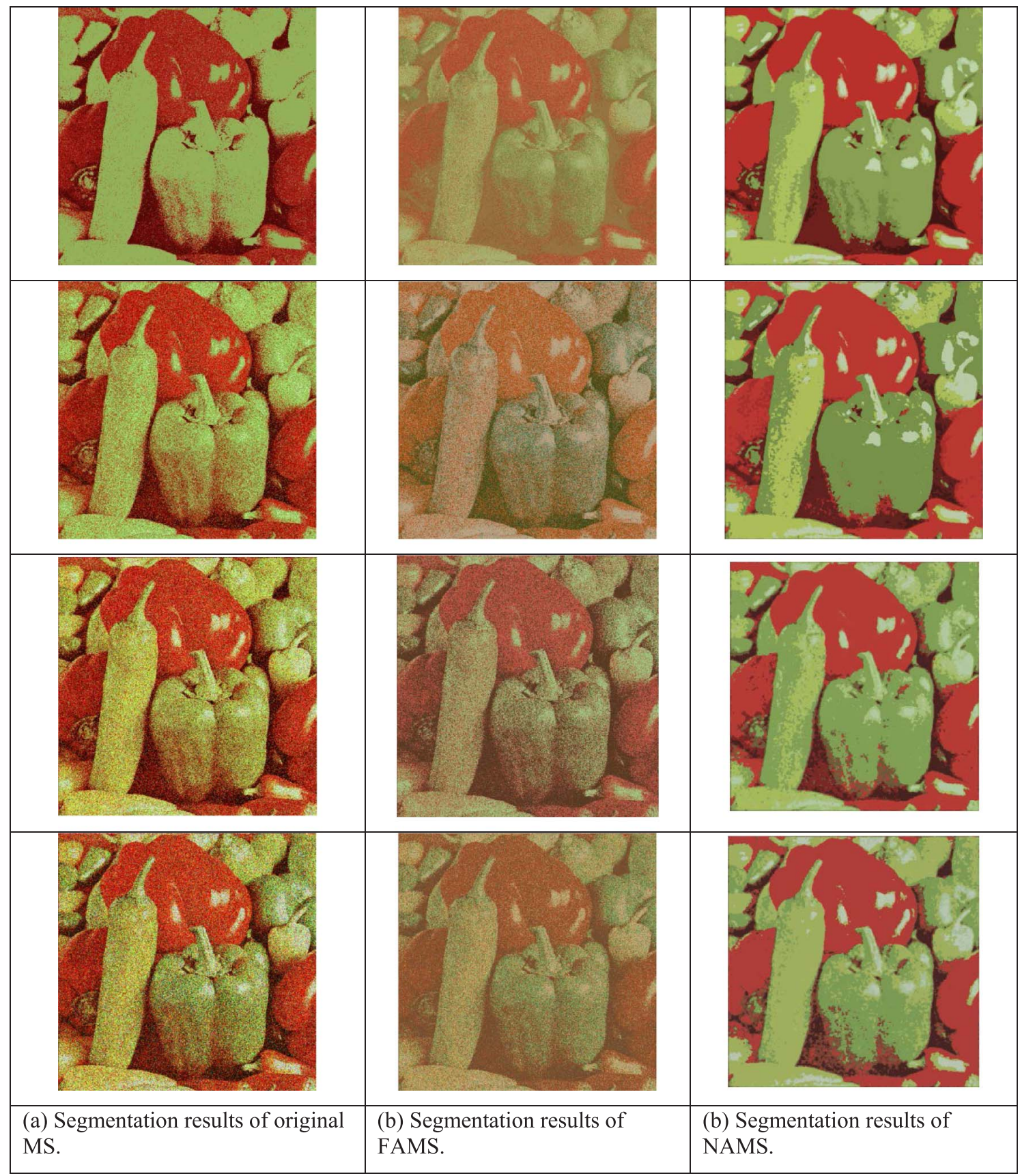

Fig. 11. Segmentation results on Peppers image with Gaussian noise. 


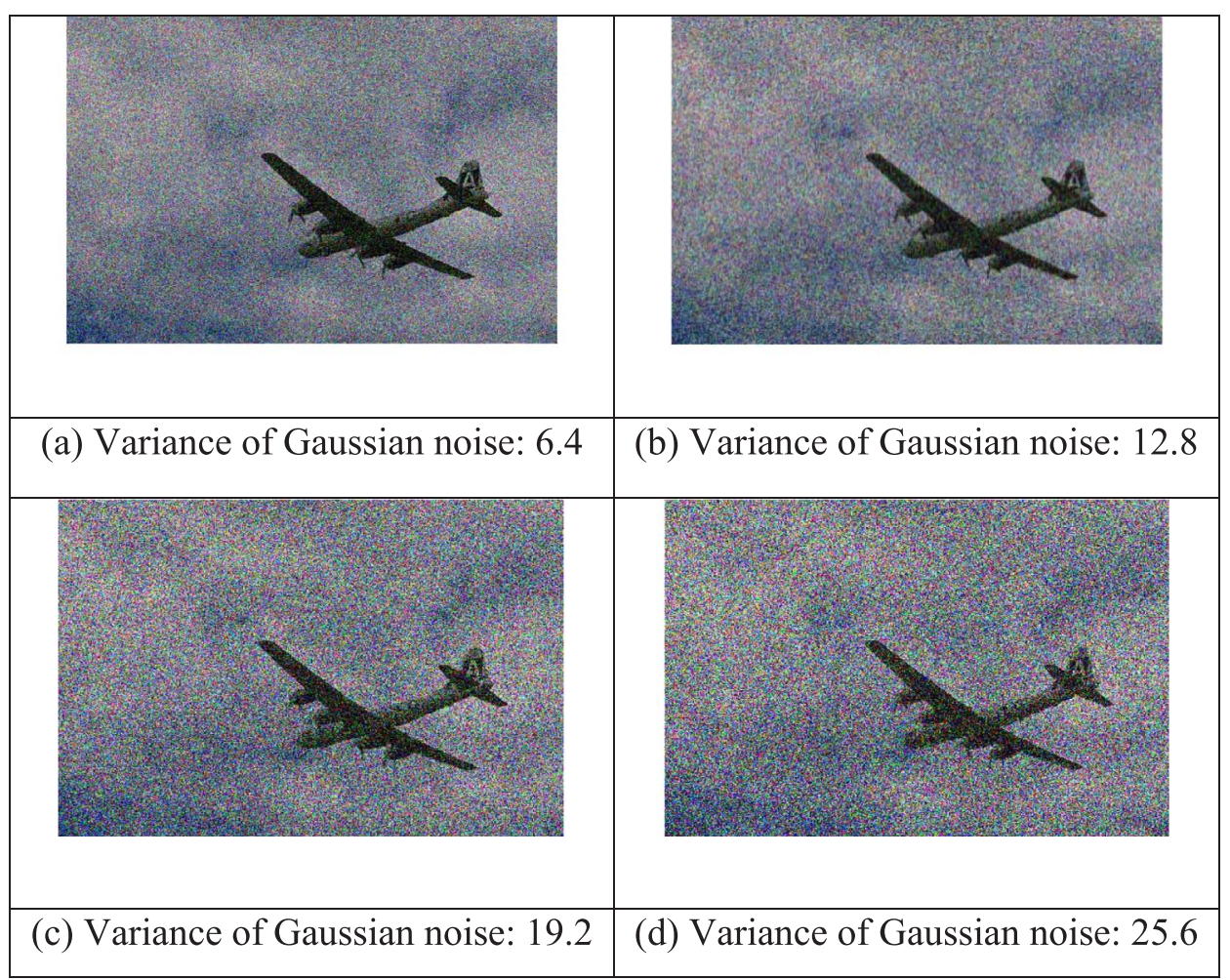

Fig.12. Plane image with Gaussian noise.

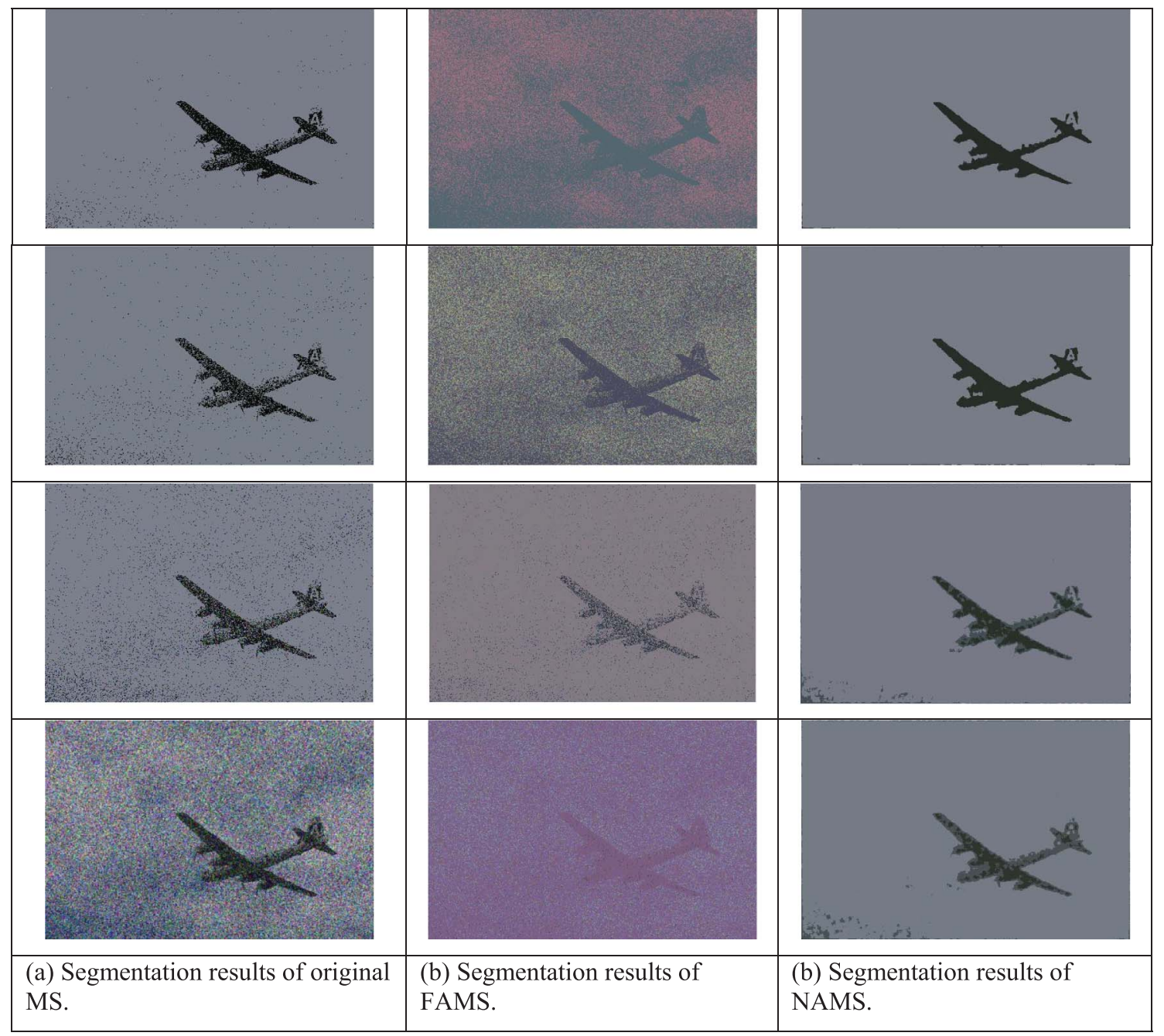

Fig. 13. Segmentation results on Plane image with Gaussian noise. 


\section{References}

[1] H.D. Cheng, X.H. Jiang, Y. Sun, J. Wang, Color image segmentation: advances and prospects, Pattern Recognit. 34 (12) (2001) 2259-2281.

[2] A. Gillet, L. Macaire, C. Botte-Lecocq, and J. G. Postaire, "Color image segmentation by analysis of 3D histogram with fuzzy morphological filters," in Springer-Verlag Editor, Fuzzy Filters for Image Processing Studies in Fuzziness and Fuzziness and Soft Computing, New York, USA, 2002, pp. 154-177.

[3] L. Shafarenko, M. Petrou, J.V. Kittler, Histogram based segmentation in a perceptually uniform color space, IEEE Trans. Image Process 7 (9) (1998) 1354-1358.

[4] C. Li, C. Xu, C. Gui, M.D. Fox, "Level set evolution without re-initialization: a new variational formulation", in Proc, San Diego, USA, IEEE Conf. Computer Vision and Pattern Recognition, 2005, pp. 430-436.

[5] W.B. Tao, H. Jin, Y.M. Zhang, "Color image segmentation based on mean shift and normalized cuts, IEEE Transact. Syst., Man, and Cybernet.—Part B: Cybernet. 37 (5) (2007) 1382-1389.

[6] R.O. Duda, P.E. Hart, D.G. Sork, Pattern Classification, Wiley-Interscience, New York, 2000.

[7] K. Haris, S.N. Efstratiadis, N. Maglaveras, A.K. Katsaggelos, Hybrid image segmentation using watersheds and fast region merging, IEEE Trans. Image Process. 7 (12) (Dec. 1998) 1684-1699.

[8] L. Vincent, P. Soille, Watersheds in digital spaces: an efficient algorithm based on immersion simulation, IEEE Trans. Pattern Anal. Mach. Intell. 13 (6) (Jun. 1991) 583-597.

[9] S. Makrogiannis, G. Economou, S. Fotopoulos, "A, region dissimilarity relation that combines feature-space and spatial information for color image segmentation", IEEE Trans. Syst., Man, Cybern. B, Cybern., Feb. 35 (1) (2005) 44-53.

[10] Z.-Y. Wu, R. Leahy, An optimal graph theoretic approach to data clustering: Theory and its application to image segmentation, IEEE Trans. Pattern Anal. Mach. Intell. 15 (11) (Nov. 1993) 1101-1113.

[11] J. Shi, J. Malik, Normalized cuts and image segmentation, IEEE Trans. Pattern Anal. Mach. Intell. 22 (8) (Aug. 2000) 888-905.

[12] Y.Z. Cheng, Mean Shift, mode seeking, and clustering, IEEE Transact. Pattern Analys. Mach. Intelligence 17 (8) (1995) 790-799.

[13] G. Domingues, H. Bischof, and R. Beichel, Fast 3D mean shift filter for CT images, in: in Proc. Scandinavian Conference On Image Analysis, Sweden, 2003, pp.
$438-445$

[14] C.J. Yang, R. Duraiswami, L. Davis, "Efficient meanshift tracking via a new similarity measure", in Proc, San Diego, USA, IEEE Conf. Computer Vision and Pattern Recognition, 2005, pp. 176-183.

[15] D. Comaniciu, P. Meer, Meanshift: a robust approach toward feature space analysis, IEEE Trans Pattern Analysis Mach. Intelligence 24 (5) (2002) 603-619.

[16] Comaniciu, Dorin, Visvanathan Ramesh, and Peter Meer, Real-time tracking of nonrigid objects using mean shift, Computer Vision and Pattern Recognition, 2000. Proceedings. IEEE Conference on. Vol. 2. IEEE, 2000.

[17] U. Ozertem, D. Erdogmus, R. Jenssen, Mean shift spectral clustering, Pattern Recognit. 41 (6) (2008) 1924-1938.

[18] W.B. Tao, J. Liu, Unified mean shift segmentation and graph region merging algorithm for infrared ship target segmentation, Opt. Eng. 46 (2007) 12.

[19] J.H. Park, G.S. Lee, S.Y. Park, Color image segmentation using adaptive mean shift and statistical model-based methods, Comput. Math. Appl. 57 (2009) 970-980.

[20] Ling-xing Liu, Guan-zheng Tan, M.Sami Soliman, Color image segmentation using mean shift and improved ant clustering, J. Central South University 19 (4) (2012) 1040-1048.

[21] Hong Liu, Yu. Ze, Hongbin Zha, Yuexian Zou, Lin Zhang, Robust human tracking based on multi-cue integration and mean-shift, Pattern Recognit. Lett. 30 (2009) (2009) 827-837.

[22] Leo Ai, Jinhu Xiong, Temporal-spatial mean-shift clustering analysis to improve functional MRI activation detection, Magnet. Resonance Imaging 34 (2016) 1283-1291.

[23] Yu-Ren Lai, Kuo-Liang Chung, Chyou-Hwa Chen, Guei-Yin Lin, Chao-Hsin Wang, Novel mean-shift based histogram equalization using textured regions, Expert Syst. Applicat. 39 (2012) 2750-2758.

[24] Mustafa Ozden, Ediz Polat, A color image segmentation approach for content-based image retrieval, Pattern Recognit. 40 (2007) 1318-1325.

[25] Keinosuke Fukunaga, Larry D. Hostetler, The estimation of the gradient of a density function, with applications in pattern recognition, IEEE Transact. Informat. Theory. IEEE. 21 (1) (1975) 32-40, http://dx.doi.org/10.1109/TIT.1975.1055330. Retrieved 2008-02-29.

[26] Xinhong Zhanga, Yanbin Cui, Duoyi Li, Xianxing Liu, Fan Zhang, An adaptive mean shift clustering algorithm based on locality-sensitive hashing, Optik - Internat. J. Light Electron Opt. 123 (20) (2012) 1891-1894. 